\title{
Differenzialdiagnose: Kawasaki-Syndrom*
}

\author{
Differential Diagnosis: Kawasaki’s Disease
}

\author{
Autor \\ M. Albani \\ Institut \\ Klinik für Kinder und Jugendliche - HSK, Wiesbaden
}

\section{Bibliografie}

DOI 10.1055/s-0029-1214590

Online-Publikation: 30. 6. 2009

Akt Dermatol 2009; 35:

331-333 @ Georg Thieme

Verlag KG Stuttgart · New York ISSN 0340-2541

Korrespondenzadresse Prof. Dr. Michael Albani Klinik für Kinder und Jugendliche - HSK Ludwig-Erhard-Straße 100 65199 Wiesbaden michael.albani@ hsk-wiesbaden.de

\section{Zusammenfassung \\ $\nabla$}

Das Kawasaki-Syndrom, erstbeschrieben durch Tomisaku Kawasaki 1967, ist eine hochfieberhafte Systemerkrankung in Form einer Vaskulitis, die zwar zahlreiche Gefäßregionen betreffen kann, insbesondere aber durch aneurysmatische Veränderungen der Koronargefäße als schwerwiegende Verlaufskomplikation gekennzeichnet ist. Klinisch imponieren die Kinder - Prädilektionsalter 2 - 5 Jahre - durch anhaltend (> 5 Tage) hohes, antibiotikaresistentes Fieber, eine ausgeprägte, auch hämorrhagische Konjunktivitis, ausgedehnte, nicht eitrige zervikale Lymphknotenschwellungen, eine Himbeerzunge, düsterrote Lacklippen, ein morbilliformes Exanthem und

\section{Klinisches Bild \\ $\nabla$}

Hohes, über mehr als 5 Tage persistierendes, antibiotikaresistentes Fieber, begleitet von hohen Entzündungszeichen (CRP, BSG) und Blutbildveränderungen mit neutrophiler Leukozytose und z.T. ausgeprägter Thrombozytose, Himbeerzunge, düsterrote Lacklippen, konfluierendes morbilliformes Exanthem und ein Palmarerythem sowie z.T. eindrucksvolle Lymphknotenschwellungen bei deutlichem Krankheitsgefühl bestimmen das klinische Bild ( $\bullet$ Abb. 1-3).

EKG-Veränderungen mit Rhythmusstörungen und Störungen der Erregungsausbreitung und -rückbildung sowie vor allem aneurysmatische Veränderungen der Koronararterien sind bereits Zeichen eines schwereren Verlaufs, der durchaus auch in einem Infarktgeschehen resultieren kann. Aber auch andere Organsysteme und Gefäßregionen (z.B. Bauchaorta) können betroffen sein und mit ausgedehnten Aneurysmen und in- ein variabel deutlich ausgeprägtes Palmar- und Plantarerythem, dem eine akral betonte, aber auch die gesamten Handflächen und Fußssohlen betreffende Hautabschilferung folgt. Je nach Betonung der Symptomatik werden die Kinder ggf. auch dem Dermatologen oder Augenarzt vorgestellt, denen dieses Krankheitsbild daher bekannt sein sollte. Eine umgehend eingeleitete Therapie mit Immunglobulinen und Azetylsalizylsäure trägt signifikant zu einer Reduktion der z.T. schwerwiegenden vaskulären Komplikationen bei. Bei Überstehen der akuten Krankheitsphase ist die Prognose in Abhängigkeit von der Koronarbeteiligung mit restloser Rückbildung der vaskulitischen Veränderungen im Allgemeinen gut.

* Vortrag beim 2. Wiesbadener Symposium Kinderdermatologie, 12. November 2008.

traparenchymatösen Veränderungen (z. B. Leber), entzündlichen Ödemen (z.B. Pankreas) und hämorrhagischen Diarrhoen einhergehen [1].

Die Diagnose ist nach Ausschluss anderer Ursachen als gesichert anzusehen, wenn außer dem Fieber fünf der genannten Kriterien vorliegen:

- Persistierendes, antibiotikaresistentes Fieber ohne erkennbare Ursache

- Zervikale, nicht eitrige Lymphknotenschwellungen ( $>1,5 \mathrm{~cm}$ Durchmesser)

- Konjunktivitis

- Morbilliformes, polymorphes Exanthem

- Lacklippen, Himbeerzunge

- Hauterscheinungen; Palmar- u. Plantarerythem mit nachfolgender lamellöser Schuppung an Fingern und Zehen

Dabei ist daran zu denken, dass mehr als ein Viertel der Verläufe mit der Entwicklung von Aneurysmen die Kriterien nicht erfüllen [2]. 


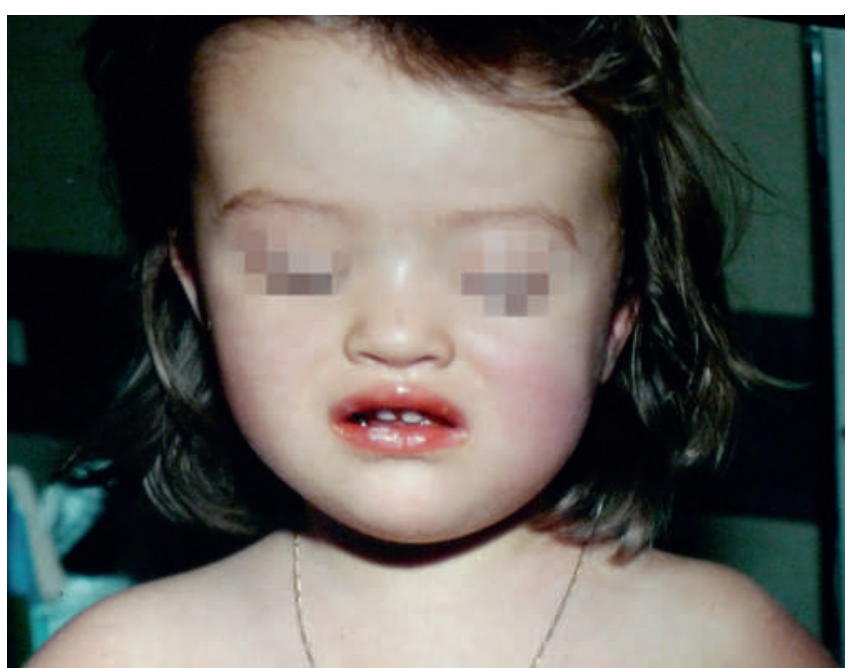

Abb. 12 Jahre und 10 Monate altes Kind mit Kawasaki-Syndrom: Exanthem, Lacklippen, schweres Krankheitsgefühl.

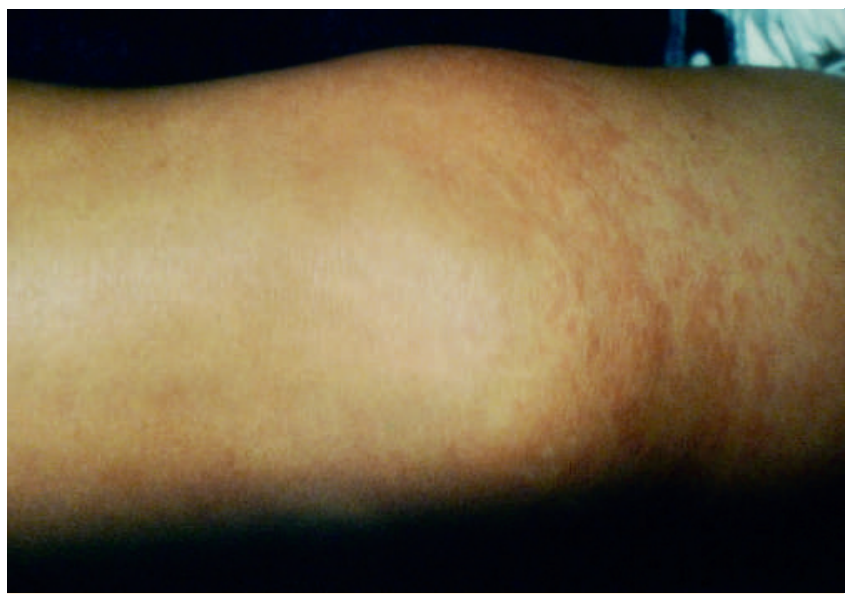

Abb. 2 Morbilliformes Exanthem am gesamten Integument (Knie).

\section{Therapie \\ $\nabla$}

Die Therapie besteht in der i.v.-Gabe von Immunglobulinen in einer Dosis von $2 \mathrm{~g} / \mathrm{kg} \mathrm{KG}$ über mehrere Stunden begleitet von ASS in einer Dosis von 60-100 mg/kg KG pro Tag. Nach Entfieberung, die bei Therapieerfolg überwiegend bereits in den nächsten 48-72 Stunden eintritt, kann die ASS-Dosis auf 3-5 mg/kg KG reduziert werden.

Bestehen nach 6 Wochen keine erhöhten Entzündungszeichen (CRP, BSG, Thrombozytose, Aneurysmen) kann die Therapie mit ASS beendet werden, zumal deren bedeutsamer Therapieanteil nach neueren Daten fraglich erscheint [3]. Davon abweichend ist eine langfristige Thrombozyten-Aggregation hemmende Therapie bei Vorliegen von Koronaraneurysmen als unverzichtbar empfohlen.

Die klinischen Symptome bilden sich auch ohne Therapie zurück mit schrittweiser Entfieberung und Schuppung der Haut sowie dem Auftreten von Koronaraneurysmen, die bei früh- und initial korrekt behandelten Kinder nicht auftreten sollten.

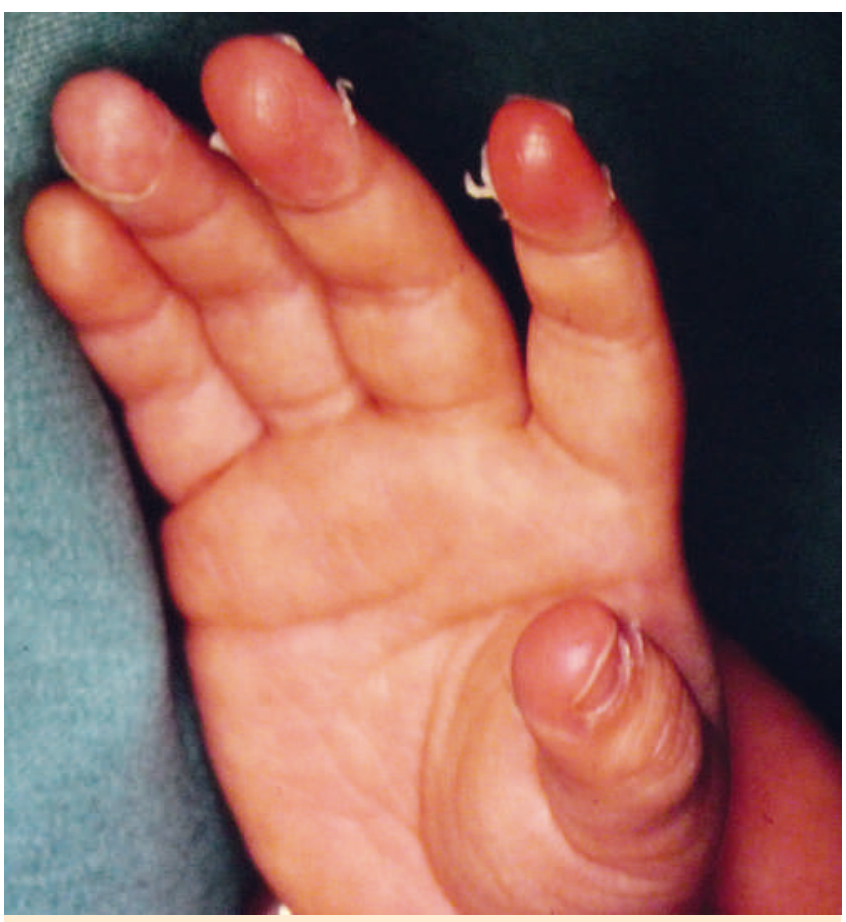

Abb. 3 Palmarerythem, abschilfernde Haut an den Akren.

\section{Epidemiologische Daten \\ $\nabla$}

In der Klinik für Kinder und Jugendliche Wiesbaden wurden in den Jahren 2000-2007 53 Fälle von Kawasaki-Syndrom diagnostiziert und behandelt. Das mittlere Erkrankungsalter lag bei 3,5 Jahren (Bereich 2 Monate - 10,1 Jahre) und der Anteil der Kinder zwischen 1 - 5 Jahren betrug $83 \%$. In 9 Fällen (17\%) zeigten sich Koronarveränderungen mittleren bis schweren Grades, die allerdings trotz früher Standardtherapie in 2 Fällen (3,7\%) persistierten. Allerdings variierte die Zeitdauer bis zur Einweisung und Behandlung zwischen 3-10 Tagen, ohne dass sich in dieser Gruppe bei den wenigen Fällen mit Koronarbeteiligung eine Korrelation zwischen späterer Behandlung und Verlauf ergab.

In der Literatur [1] ist dies jedoch wiederholt belegt, sodass eine frühe Diagnose und Therapie von unbestritten prognostischer Bedeutung sind.

\section{Differenzialdiagnosen \\ $\nabla$}

Daher ist eine genaue Kenntnis der differenzialdiagnostischen Alternativen wichtig.

Diese sind in der Tab. 1 wiedergegeben und sollen nur auszugsweise diskutiert werden.

Lymphknotenschwellungen, morbilliformes Exanthem und Himbeerzunge werden schnell den Verdacht auf das Vorliegen einer Scharlacherkrankung ergeben, die jedoch mit dem Nachweis stets Antibiotika-sensibler A-Streptokokken binnen weniger Tage erfolgreich zu behandeln ist.

Der typische absteigende Verlauf des Exanthems, der Befund von Koplik'schen Flecken während der ersten Fieberphase und der Nachweis eines Masern-IgM wird die durchaus mögliche Differenzialdiagnose einer Masernerkrankung schnell verifizieren lassen. 


\section{Tab. 1 DD Kawasaki-Syndrom.}

Differenzialdiagnose des Kawasaki-Syndroms
Scharlach
Masern
Toxic-Schock-Syndrom
Erythema exsudativum multiforme
Medikamenten-Hypersensitivitäts-Reaktion (SJS)
idiopathische juvenile Arthritis
Adeno-Virus-Infektion
Gianotti-Crosti-Syndrom
RM-spotted Fieber
Leptospirose

Schwieriger fällt die Differenzialdiagnose bei der medikamenten-toxischen Reaktion oder dem Erythema exsudativum multiforme. Doch wird eine einschlägige Medikamentenanamnese, das Fehlen jeglicher Koronarbeteiligung sowie die vorausgehende fieberhafte Erkrankung die Entscheidung erleichtern.

Andere ( Tab.1) in Teilen ihrer klinischen Symptomatik (Lymphknotenschwellungen, Exanthem, konjunktivale Injektionen) ähnliche Krankheitsbilder werden sich durch weniger hochfieberhaften Verlauf, die doch bei näherem Hinsehen Andersartigkeit des Exanthems oder durch das weniger schwere Krankheitsbild und letztlich natürlich durch das Fehlen einer koronaren Beteiligung abgrenzen lassen. Das stets differenzialdiagnostisch in Betracht zu ziehende Kawasaki-Syndrom ist eine wichtige Aufgabe.

Dies umso mehr, als es sich um eine rein klinische Diagnose handelt, für die kein spezifischer, die Diagnose sichernder hämatologischer, serologischer oder biochemischer Parameter zur Verfügung steht. Zwar ist es allgemeiner Konsens, dass die Erkrankung durch ein infektiöses Agens ausgelöst wird, das jedoch bisher nicht hat identifiziert werden können. Neutrophilen-Infiltration, gefolgt von Makrophagen, CD8-T-Lymphozyten und vornehmlich IgA-Plasmazellen kennzeichnen die Inflammation der Gefäße. Die Vaskulitis kann alle drei Schichten der Wand betreffen mit Auflösen der strukturellen Integrität, woraus die Erweiterungen und aneurysmatischen Aussackungen resultieren. Darüber hinaus sind auch nicht vaskuläre Gewebe wie Myokard, Lunge, Pankreas und Niere von IgA-Plasmazellinfiltraten betroffen, sodass dies die Frage aufwirft, ob der direkte inflammatorische Effekt oder die immunologische Abwehrreaktion des Organismus für diese Erscheinungen verantwortlich ist.

Die zudem familiär und in bestimmten ethnischen Gruppen gehäuft vorkommende Erkrankung lässt eine genetische Empfänglichkeit als Grundlage vermuten. Dazu konnten Breunis et al. [4] zeigen, dass in den allgemeinen Chemokin-Rezeptor-Gen-Vari- anten unter den Einzel-Nucleotid-Polymorphismen (SNPs) im CCR3-CCR2-CCR5-Gen-Cluster vier die Empfänglichkeit für das Kawasaki-Syndrom signifikant erhöht ist. Ob sich aus diesen molekulargenetischen Untersuchungsbefunden eine die Diagnose sichernde Bedeutung entwickeln wird, ist derzeit nicht $\mathrm{zu}$ sagen.

\section{Abstract}

\section{Differential Diagnosis: Kawasaki's Disease $\nabla$}

Kawasaki disease, first described by Tomisaku Kawasaki in 1967 represents a febrile systemic vasculitis affecting several vascular regions, particularly the coronary arteries with aneurysmatic dilatations. The clinical picture in children - mainly between $2-5$ years of age - is dominated by continuous high fever lasting more than 5 days resistant to antibiotic treatment, hemorrhagic conjunctivitis, nonsuppurative cervical lymphadenopathy, strawberry tongue, dry cracked lips, rash of various forms, almost maculopapular or scarlattiform and edema and erythema of hands and feet with following desquamation of fingers and toes which may involve the entire hand and foot.

According to the main clinical features the children may also be first presented to dermatologist or ophthalmologist so that these specialities should be familiar with this disease.

An immediate instigation of treatment with intravenous immunoglobulines and acetylsalicylic acid contributes to a significant reduction of coronary aneurysmatic complications. Surviving the acute phase of illness recovery is almost complete and without long-term effects for those who do not develop severe coronary vascular disease.

\section{Literatur}

1 Newburger JW, Takahashi M, Gerber MA et al. Diagnosis, Treatment, and Long-Term Management of Kawasaki Disease: A Statement for Health Professionals From the Committee on Rheumatic Fever, Endocarditis, and Kawasaki Disease, Council on Cardiovascular Disease in the Young. American Heart Association Pediatrics 2004; 114: 17081733

2 Muta $\mathrm{H}$, Ishii $M$, Egami $\mathrm{K}$ et al. Early intravenous gamma-globulin treatment for Kawasaki disease: The nationwide surveys in Japan. J Pediatrics 2004; 144: 496 - 499

3 Hsieh KS, Weng KP, Lin CC, Huang TC, Lee CL, Huang SM. Treatment of Acute Kawasaki Disease: Aspirin's Role in the Febrile Stage Revisited. Pediatrics 2004; 114: 689-693

4 Breunis WB, Biezeveld MH, Geissler J, Kuipers IM et al. Polymorphisms in chemokine receptor genes and susceptibility to Kawasaki disease. Clin Exp Immunol 2007; 150: 83-90 